07

\title{
Механизм влияния дисперсных наночастиц на параметры мартенситных переходов в сплавах с эффектом памяти формы
}

\author{
(C) Г.А. Малыгин \\ Физико-технический институт им. А.Ф. Иоффре, \\ Санкт-Петербург, Россия \\ E-mail: malygin.ga@mail.ioffe.ru \\ Поступила в Редакцию 1 июля 2019 г. \\ В окончательной редакции 1 июля 2019 г. \\ Принята к публикации 2 июля 2019 г.
}

\begin{abstract}
В рамках теории размытых мартенситных переходов, базирующейся на термодинамических и кинетических уравнениях и соотношениях, анализируется механизм влияния дисперсных наночастиц на параметры мартенситных переходов в сплавах с ЭПФ. В качестве объектов анализа выбраны сплав ТіNi с вариацией размера частиц $\mathrm{Ti}_{3} \mathrm{Ni}_{4}$ при постоянной их объемной концентрации, и сплав $\mathrm{NiMnGaTb}$ с частицами выделений $\mathrm{Tb}$ постоянного размера при вариации объемной концентрации выделений. Сведения об этих сплавах имеются в литературе. Анализ показал, что из-за когерентного характера связи частиц $\mathrm{Ti}_{3} \mathrm{Ni}_{4}$ с матрицей температурная ширина $R-B 19^{\prime}$ перехода зависит от размера частиц $d$, как $1 / d$, что подтверждает ранее установленную закономерность влияния внутренних локальных напряжений на этот параметр. Что касается сплава NiMnGaTb, то анализ показал, что из-за наличия внутренних локальных напряжений, связанных с частицами $\mathrm{Tb}$, температурная ширина мартенситного перехода увеличивается линейно с ростом концентрации частиц в сплаве. Показано также существование критической величины концентрации частиц, выше которой температурная ширина перехода становится неопределенно большой, и мартенситное превращение в сплаве блокируется.
\end{abstract}

Ключевые слова: сплавы с ЭПФ, мартенситные переходы, дисперсные наночастицы, дислокации фазового превращения.

DOI: 10.21883/FTT.2019.11.48415.542

\section{1. Введение}

Сплавы с эффектом памяти формы (ЭПФ) - перспективные материалы для применения в различных высокотехнологичных областях науки и техники. Они являются в настоящее время предметом интенсивных исследований с целью улучшения их функциональных свойств. Эти свойства непосредственным образом связаны с параметрами протекающих в сплавах с ЭПФ термопругих мартенситных переходов. Наиболее важными параметрами являются температура $T_{c}$, интервал $\Delta T$ и гистерезис $\Delta T_{n}$ перехода, а также величина деформации мартенситного превращения $\varepsilon_{m}$. Согласно экспериментальным данным, рассматриваемые параметры чувствительны к структуре сплава не только на атомном уровне (содержанию и соотношению в сплаве компонентов), но и на мезо (микро и нано) уровне. Исследования показывают, что рассматриваемые параметры чувствительны к размеру кристалла сплава в целом, а в поли (микро и нано) кристаллическом материале - к размеру кристаллитов.

Другой важный фактор, влияющий на параметры мартенситных переходов в этих сплавах, это наличие в них дисперсных нано- и микрочастиц [1-6]. В сплаве $\mathrm{TiNi}$ - это частицы интерметаллида $\mathrm{Ti}_{3} \mathrm{Ni}_{4}$ [2,3], в сплаве $\mathrm{Ni}_{50-x} \mathrm{Mn}_{30} \mathrm{Ga}_{20} \mathrm{~Tb}_{x}$ - частицы выделений тербия [1], в компактированном после размола порошке сплава $\mathrm{Ti}-\mathrm{Ni}-\mathrm{Hf}$ - частицы карбида $\mathrm{HfC}$ [5], а в сплаве $\mathrm{Cu}-\mathrm{Al}-\mathrm{Ni}$ - частицы двуокиси $\mathrm{HfO}_{2}$ [6]. Влияние частиц, как показывают эксперименты, зависит от размера частиц, их объемной плотности, а также от наличия или отсутствия у частиц когерентной связи с матрицей. Поскольку частицы являются одновременно препятствиями для перемещения решеточных дислокаций, то их наличие в сплаве с ЭПФ расширяет рабочий диапазон напряжений использования сплава в различных силовых и сенсорных устройствах. К настоящему времени накоплен достаточно большой объем данных по влиянию дисперсных нано и микрочастиц на параметры мартенситных переходов в сплавах с ЭПФ. Но их теоретическое осмысление не выходит за рамки качественного рассмотрения вопроса ввиду отсутствия структурно чувствительной на мезоуровне теории фазовых переходов первого рода, к которым относятся мартенситные переходы в рассматриваемых сплавах. Это не позволяет количественно установить явную зависимость параметров мартенситного перехода в каждом конкретном сплаве от размера и объемной плотности частиц и влияние на эти параметры когерентной связи частиц с матрицей или ее отсутствия.

Наиболее близкой к такой структурно чувствительной на мезоуровне теории мартенситных переходов в сплавах с ЭПФ является в настоящее время теория размытых мартенситных переходов (РМП) [7-9]. Она 
уже использовалась для анализа размерных эффектов в этих сплавах и позволила установить явную зависимость параметров мартенситных переходов в ряде сплавов с ЭПФ от поперечного сечения кристалла $D$, а в поликристаллическом материале - от размера кристаллитов $d$ в диапазоне размеров $D, d=10-1000 \mathrm{~nm}[8,9]$. В частности, анализ показал, что в отсутствие внутренних локальных микронапряжений зависимость температурного интервала (размытия) перехода $\Delta T$ от размерных факторов $D$ или $d$ имеет вид $\Delta T \sim 1 / D^{2}$ или $\Delta T \sim 1 / d^{2}$, а при наличии микронапряжений, соответственно, как $\Delta T \sim 1 / D$ или $\Delta T \sim 1 / d$.

Цель настоящей работы состоит в том, чтобы на основе теории РМП осуществить аналогичный анализ относительно влияния дисперсных нано и микрочастиц, их размера $d$ и относительной объемной плотности $f$, а также когерентной связи с матрицей на параметры мартенситных переходов в сплавах $\mathrm{Ni}_{50-x} \mathrm{Mn}_{30} \mathrm{Ga}_{20} \mathrm{~Tb}_{x}$ [1] и TiNi [3]. Указанные сплавы интересны для анализа тем, что в [1] варьируется объемная плотность частиц Ть при постоянном их размере, а в [3], наоборот, варьируется размер частиц $\mathrm{Ti}_{3} \mathrm{Ni}_{4}$ при постоянной их концентрации.

\section{2. Основные соотношения теории РМП}

Теория размытых мартенситных переходов базируется как на термодинамических, так и на кинетических соотношениях. Основное термодинамическое соотношение в случае одностадийного мартенситного перехода имеет вид $[7,8]$

$$
\varphi_{M}=\frac{1}{1+\exp \left(\Delta U / k_{\mathrm{B}} T\right)}, \quad \varphi_{A}=1-\varphi_{M},
$$

где $\varphi_{M}$ и $\varphi_{A}$ - относительные объемные доли мартенсита и аустенита в сплаве, $\Delta U=\omega(d, f) \Delta u$ - изменение свободной энергии сплава при образовании в нем элементарного объема новой фазы $\omega_{d, f}$, ограниченного (стесненного, constrained) структурными факторами $d$ и $f$,

$$
\frac{1}{\omega(d, f)}=\frac{1}{\omega_{0}}+\frac{1}{\omega_{d, f}}
$$

$\omega_{0}$ - элементарный объем превращения в отсутствие его пространственного стеснения (constraint) частицами,

$$
\Delta u=q \frac{T-T_{c}}{T_{c}}-\varepsilon_{m} \sigma+W\left(\sigma_{e}\right),
$$

$\Delta u$ - объемная плотность свободной энергии мартенситного перехода, $q$ - теплота перехода, $T$ - температура, $T_{c}$ - температура равенства объемов мартенситной и аустенитной фаз в сплаве, $\varepsilon_{m}$ - деформация мартенситного превращения, $\sigma$ - механическое напряжение, приложенное к кристаллу, $W\left(\sigma_{e}\right)=\varepsilon_{m} \sigma_{e}$ энергия внутренних упругих напряжений $\sigma_{e}$ из-за наличия когерентной связи частиц с решеткой кристалла, $k_{\mathrm{B}}-$ постоянная Больцмана. Явная зависимость элементарного объема превращения $\omega_{d, f}$ от размера частиц $d$ и их относительной объемной плотности $f=\left(\beta d / l_{0}\right)^{3}$ определяется соотношениями

$$
\begin{gathered}
\omega_{d, f}=l_{p}^{2}(d, f) a_{0}, \quad l_{p}=\left(l_{0}-\beta d\right)^{2} / \beta d=\beta R(f) d, \\
R(f)=\left(\frac{1-f^{1 / 3}}{f^{1 / 3}}\right)^{2},
\end{gathered}
$$

где $l_{p}$ - расстояние свободного пробега дислокаций превращения до встречи их с частицами, $\beta=\left(\pi \beta_{0} / 4\right)^{1 / 3}$, $\beta_{0}=h / d=\mathrm{const}-$ коэффициент формы частицы в виде диска высотой $h$ и диаметром $d \gg h, a_{0}-$ расстояние между габитусными плоскостями в сплаве. Кроме препятствий для перемещения дислокаций фазового превращения, дисперсные частицы являются источником гетерогенного зарождения этих дислокаций. Согласно [9] при гетерогенном зарождении дислокаций превращения элементарный объем превращения $\omega$ равен $\left(\pi a^{2} / 4 \varepsilon_{m}\right) \beta d$. В результате, для полного элементарного объема превращения получаем соотношение

$$
\frac{1}{\omega(d, f)}=\frac{1}{\omega_{0}}\left[1+\frac{\omega_{0}}{\omega_{d, f}}\right]=\frac{1}{\omega_{0}}\left[1+\left(\frac{d_{0}}{\beta R(f) d}\right)^{2}\right],
$$

где $d_{0}=\omega_{0}^{1 / 3} \sim d^{1 / 3}, a-$ параметр решетки.

Кинетические соотношения для параметров мартенситного перехода находятся из решения кинетических уравнений для объемных долей мартенситной $\varphi_{M}$ и аустенитной $\varphi_{A}$ фаз $[7,8]$. В [8] приведено частное решение этих уравнений в случае равенства объемных долей мартенсита и аустенита (равенства размеров мартенситных и аустенитных ламелей) в кристалле сплава. Это решение определяет температуры $T_{c}$, соответственно, при прямом $T_{c}^{M}$ и обратном $T_{c}^{A}$ мартенситных переходах. В сплаве с дисперсными частицами указанные температуры следующим образом зависят от структурных факторов $d$ и $f$ (при напряжении $\sigma=0$ в (1c))

$$
\begin{gathered}
T_{c}^{M}=\left[1-\frac{W\left(\sigma_{e}\right)}{q}+\frac{k_{\mathrm{B}} T_{c 0}}{q \omega(d, f)} \ln \left[\frac{3}{2} k_{0}(d, f)-1\right]\right] T_{c 0}, \\
T_{c}^{A}=\left[1+\frac{k_{\mathrm{B}} T_{c 0}}{q \omega(d, f)} \ln \left[3 k_{0}(d, f)-1\right]\right] T_{c 0} .
\end{gathered}
$$

Разница между ними $\Delta T=T_{c}^{A}-T_{c}^{M}-$ ширина (размытие) перехода по температуре,

$$
\Delta T=\left[\frac{W\left(\sigma_{e}\right)}{q}+\frac{k_{\mathrm{B}} T_{c 0}}{q \omega(d, f)} \ln \left(\frac{3 k_{0}(d, f)-1}{3 k_{0}(d, f) / 2-1}\right)\right] T_{c 0},
$$

$T_{c 0}$ - температура равенства объема фаз в отсутствие внутренних напряжений и пространственного стеснения превращения,

$$
k_{0}(d, f)=\left(\frac{l_{p}}{d_{0}}\right)^{2}=\left(\frac{d}{d_{0}} \beta R(f)\right)^{2},
$$

Соотношение $(3 \mathrm{~d})$ - вклад кинетического фактора в структурный аспект мартенситного перехода. При записи уравнения (3b) учтено отсутствие в сплаве внутренних напряжений при обратном мартенситном переходе. 


\section{3. Анализ экспериментальных данных}

\section{1. Сплав TiNi}

Особенностью сплава является то, что при содержании никеля более $50.3 \mathrm{at} \%$ его отжиг в течение $1-1.5 \mathrm{~h}$ при температурах 700-800 K приводит к образованию в сплаве дискообразных наночастиц интерметаллида $\mathrm{Ti}_{3} \mathrm{Ni}_{4}$, служащих источниками внутренних напряжений и формирования ромбоэдрического $(R)$ мартенсита. В результате, одностадийный $B 2-B 19^{\prime}$ переход становится двухстадийным $B 2-R-B 19^{\prime}$. При этом, обратный переход имеет практически одностадийный характер изза близости температур $B 19^{\prime}-R$ и $R-B 2$ переходов [10].

На рис. 1 приведены зависимости температур начала $M_{s}$ и конца $M_{f}$ прямого $R-B 19^{\prime}$, и начала $A_{s}$ и конца $A_{f}$ обратного $B 19^{\prime}-B 2$ мартенситных переходов от размера частиц $\mathrm{Ti}_{3} \mathrm{Ni}_{4}$, полученные при калориметрическом исследовании [3] кристаллов сплава $\mathrm{TiNi}$ c 50.7 at\% Ni. Обращают на себя внимание два обстоятельства. Это большая температурная ширина прямого мартенситного перехода $\Delta T^{M}=M_{s}-M_{f}$ в диапазоне размеров $d<100 \mathrm{~nm}$ по сравнению с шириной обратного перехода $\Delta T^{A}=A_{f}-A_{s}$ и наличие критического размера частиц $d_{k}=35 \mathrm{~nm}$. При размерах частиц $d<100 \mathrm{~nm}$ температуры начала и конца мартенситных превращений начинают резко снижаться и при $d_{k}=35 \mathrm{~nm}$ устремляются к нулю.

На рис. 2 приведена зависимость температур $T_{c}$ при прямом, $T_{c}^{M}=\left(M_{s}+A_{f}\right) / 2, \quad$ и обратном, $T_{c}^{A}=\left(M_{f}+A_{s}\right) / 2$, мартенситных переходах от размера частиц интерметаллида согласно приведенным на рис. 1 данным [3]. На рис. 2 кривые 1 и 2 демонстрируют результаты расчета этих температур согласно

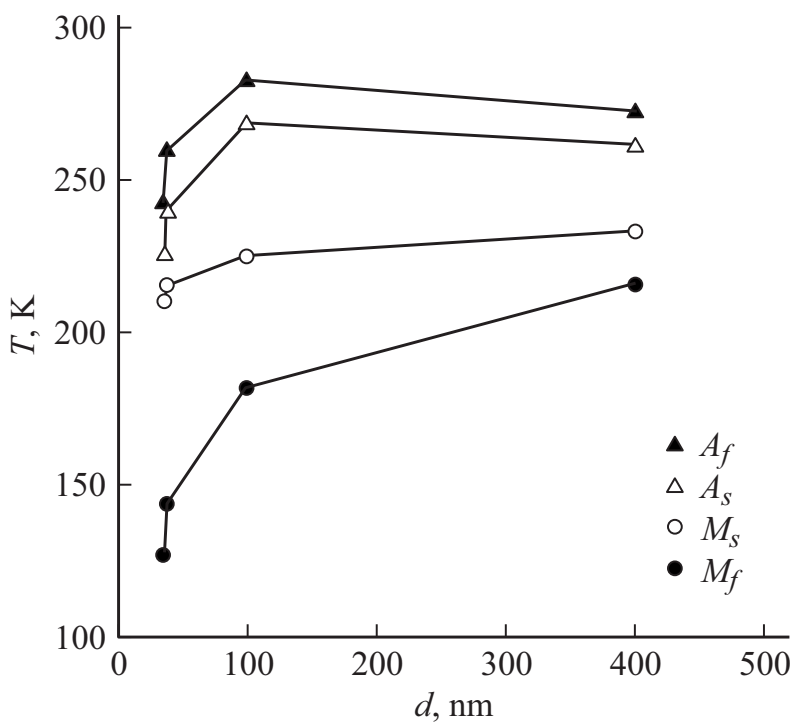

Рис. 1. Зависимость температур начала $M_{s}$ и конца $M_{f}$ прямого, и начала $A_{s}$ и конца $A_{f}$ обратного мартенситного перехода $R-B 19^{\prime}$ в кристаллах сплава TiNi с концентрацией никеля $50.7 \%$ от размера частиц $\mathrm{Ti}_{3} \mathrm{Ni}_{4}$ [3].

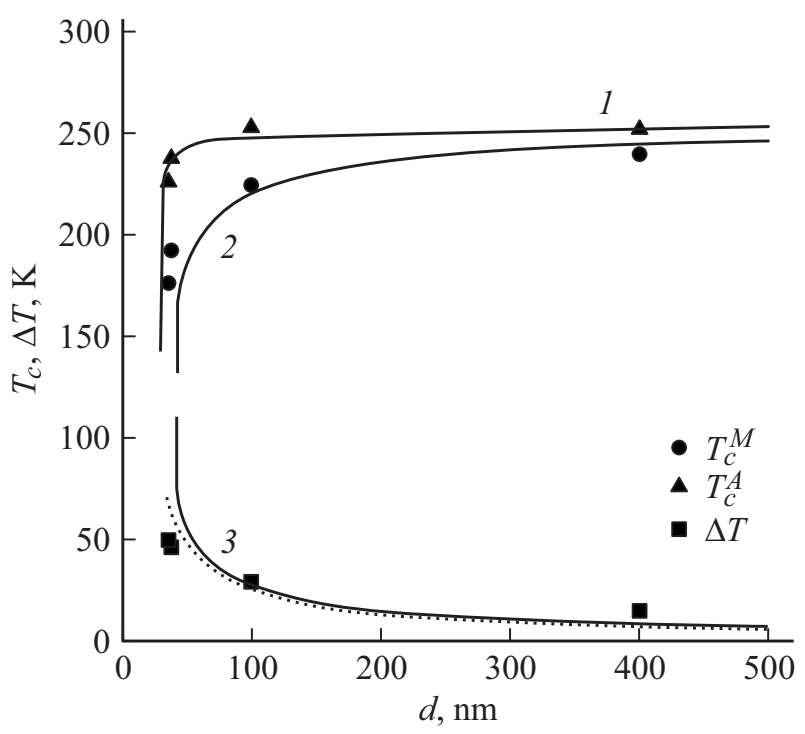

Рис. 2. Зависимость температур равного объема фаз при прямом $T_{c}^{M}=\left(M_{s}+A_{f}\right) / 2$ и обратном $T_{c}^{A}=\left(A_{s}+M_{f}\right) / 2$ мартенситных переходах в кристаллах сплава TiNi с концентрацией никеля $50.7 \%$ от размера частиц $\mathrm{Ti}_{3} \mathrm{Ni}_{4}$ [3]. Кривые 1 и 2 расчет согласно уравнениям (3b) (кривая 1) и (5b) (кривая 2) и (кривая 3). Пунктир - вклад внутренних напряжений в температурную ширину $R-B 19^{\prime}$ перехода согласно уравнению $(5 \mathrm{c})$.

уравнениям (3a) и (3b) при величине $k T_{c 0} / \omega_{0} q=10^{-2}$, $f=4 \%, R(f)=3.7, \beta_{0}=0.08, \beta=0.34, d_{k}=35 \mathrm{~nm} \mathrm{и}$ $d_{0}=65 \mathrm{~nm}$. Согласно этим уравнениям при величине кинетического фактора $k_{0}\left(d_{k}, f\right)=1 / 3$ и $2 / 3$ величина логарифмов становится равной минус бесконечности, а температуры $T_{c}^{M}$ и $T_{c}^{A}-$ равными нулю. В результате, температурная ширина перехода $\Delta T=T_{c}^{A}-T_{c}^{M}$ оказывается неопределенно большой (кривая 3 ). При расчете кривой 2 согласно уравнению (3a) учтено влияние упругой энергии внутренних напряжений $W_{e}=\varepsilon_{m} \sigma_{e}$ на температуру $T_{c}^{M}$ (см. ниже уравнения (7)). Согласно Эшелби $[11,12]$ (см. также [13]) упругая деформация $\varepsilon_{z}$ вне преципитата в виде тонкого диска толщиной $h$ и диаметром $d$, помещенного в полость диаметром $d$ и высотой $h_{0}<h$ с деформацией $\delta_{z}=\left(h-h_{0}\right) / h_{0}$, изменяется в направлении нормальном плоскости диска $z$, как

$$
\varepsilon_{z}=\left(\frac{2-v}{1-v}\right) \frac{\delta_{z} h d^{2} z}{16\left(r^{2}+z^{2}\right)^{2}},
$$

где $r$ - радиальная координата, $v-$ коэффициент Пуассона. При $r=0$ и $z=d$ получаем согласно (4а) величину деформации $\varepsilon_{z}$ и микронапряжений $\sigma_{e}=\sigma_{z}$ вне частицы $(z>h / 2)$,

$$
\varepsilon_{z}=\left(\frac{2-v}{1-v}\right) \frac{\delta_{z} h}{8 d}, \quad \sigma_{z}=E \varepsilon_{z},
$$

где $E$ - модуль упругости сплава. Подставляя, далее, энергию внутренних напряжений $W=\varepsilon_{m} \sigma_{z}$ в уравнения (3a) и (3c), получаем зависимость температуры 


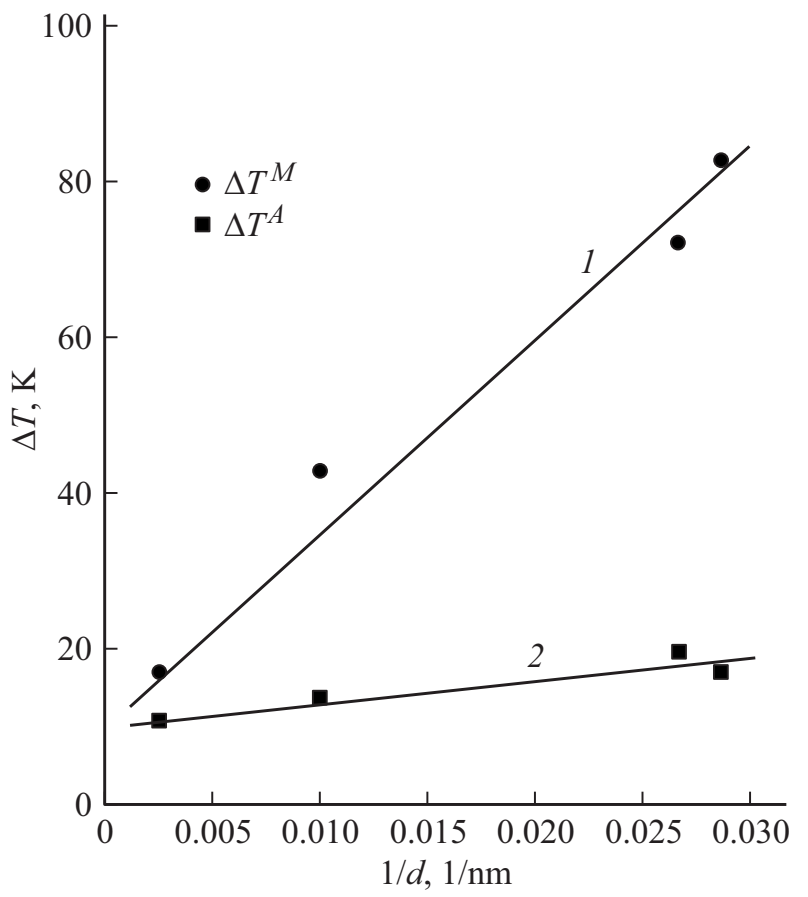

Рис. 3. Температурная ширина прямого $\Delta T^{M}$ и обратного $\Delta T^{A}$ переходов в зависимости от размера частиц $\mathrm{Ti}_{3} \mathrm{Ni}_{4}$ в координатах $\Delta T-1 / d$ в кристаллах сплава TiNi с концентрацией никеля $50.7 \%$. Экспериментальные точки - [3]. Кривые 1 и $2-$ см. текст.

$T_{c}^{M}(d)$ и температурной ширины перехода $\Delta T(d)$ от размера частиц с учетом влияния на эти параметры внутренних напряжений,

$$
\begin{gathered}
T_{c}^{M}=\left[1-\delta_{\sigma} \frac{d_{k}}{d}+\frac{k_{\mathrm{B}} T_{c 0}}{q \omega(d, f)} \ln \left[\frac{3}{2} k_{0}(d, f)-1\right]\right] T_{c 0} \\
\Delta T=\left[\delta_{\sigma} \frac{d_{k}}{d}+\frac{k_{\mathrm{B}} T_{c 0}}{q \omega(d, f)} \ln \left(\frac{3 k_{0}(d, f)-1}{3 k_{0}(d, f) / 2}-1\right)\right] T_{c 0} \\
\Delta T_{\sigma}=\delta_{\sigma} \frac{d_{k}}{d}, \quad \delta_{\sigma}=\frac{(2-v) \delta_{z} \varepsilon_{m} E}{8(1-v) q}\left(\frac{h}{d_{k}}\right)
\end{gathered}
$$

На рис. 2 кривые 2 и 3 рассчитаны согласно уравнениям (5) при величине параметра $\delta_{\sigma}=0.29$ и указанных выше значениях остальных коэффициентов и параметров. Пунктиром на рис. 2 обозначен вклад внутренних напряжений $\Delta T_{\sigma}$ в температурную ширину перехода $R-B 19^{\prime}$. Видно, что этот вклад является основным при размере частиц $d>d_{k}$. Указанное выше значение коэффициента $\delta_{\sigma}=0.29$ получено согласно соотношению (5c) при разумных значениях параметров: $\delta_{z}=3 \cdot 10^{-2}[13], \varepsilon_{m}=6 \cdot 10^{-2}, q=30 \mathrm{MJ} / \mathrm{m}^{3}[14]$, $E=150 \mathrm{GPa}[3] v=0.3$ и $h=2.8 \mathrm{~nm}$. Рис. 3 демонстрирует зависимость температурной ширины перехода при прямом и обратном мартенситном превращении в координатах $\Delta T \sim 1 / d$. Наклон прямой 1 соответствует приведенному выше значению коэффициента $\delta_{\sigma}=0.29$.
Видно, что при обратном переходе наклон прямой 2, $\delta_{\sigma}=0.034$, на порядок ниже. Таким образом, анализ подтверждает ранее установленную закономерность, что наличие в сплаве локальных упругих напряжений определяет характерный вид зависимости температурной ширины перехода, как $\Delta T(d) \sim 1 / d[9]$, где в рассматриваемом случае $d$ - размер дисперсных наночастиц.

\section{2. Сплав $\mathrm{Ni}_{50-x} \mathrm{Mn}_{30} \mathrm{Ga}_{20} \mathrm{~Tb}_{x}$}

Химический состав исследованного в [1] сплава отличается от трехкомпонентного сплава $\mathrm{Ni}_{50} \mathrm{Mn}_{30} \mathrm{Ga}_{20}$ тем, что небольшая часть атомов Ni $(x=0-1 \%)$ замещена атомами Тb. Сплав в расплавленном виде подвергался сверхбыстрой кристаллизации (melt-spinning technology), в результате чего в нем формировались зеренная структура с размером зерен $215 \mathrm{~nm}$ и выделения $\mathrm{Tb}$ в виде когерентных с матрицей частиц размером $10 \mathrm{~nm}$. С ростом концентрации тербия размеры частиц и зерен оставались постоянными, а объемная плотность частиц возрастала. Рис. 4 демонстрирует температуры равнообъемной концентрации фаз $T_{c}$ при прямом, $T_{c}^{M}$, и обратном, $T_{c}^{A}$, мартенситных переходах в сплаве $\mathrm{NiMnGaTb}$ в зависимости от объемной концентрации частиц тербия $f$. Для построения кривых использовались результаты калориметрического исследования полученных при кристаллизации лент сплава [1]. На рис. 5 показано, как изменяется усредненная величина температуры равнообъемной концентрации фаз, $\bar{T}_{c}=\left(T_{c}^{M}+T_{c}^{A}\right) / 2$, она плавно снижается с ростом концентрации тербия. В [1] найдено, что при концентрации тербия 1\% мартенситное превращение в сплаве отсутствует. По аналогии с продемонстрированным выше существованием крити-

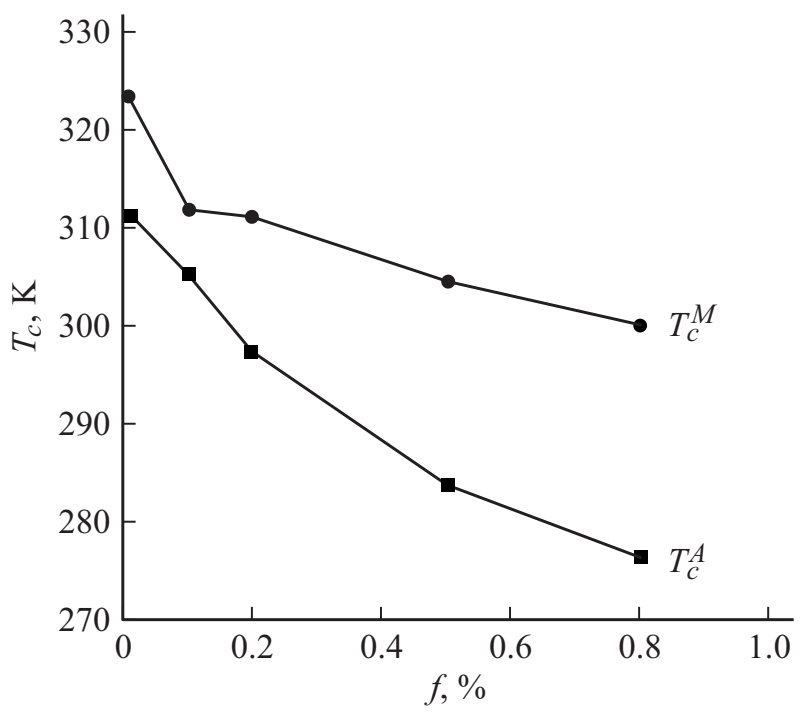

Рис. 4. Зависимость температур равной объемной концентрации мартенситной и аустенитной фаз при прямом $T_{c}^{M}=\left(M_{s}+A_{f}\right) / 2$ и обратном $T_{c}^{A}=\left(A_{s}+M_{f}\right) / 2$ мартенситных переходах в сплаве $\mathrm{Ni}_{50-x} \mathrm{Mn}_{30} \mathrm{Ga}_{20} \mathrm{~Tb}_{x}$ от концентрации частиц $\mathrm{Tb}[1]$. 


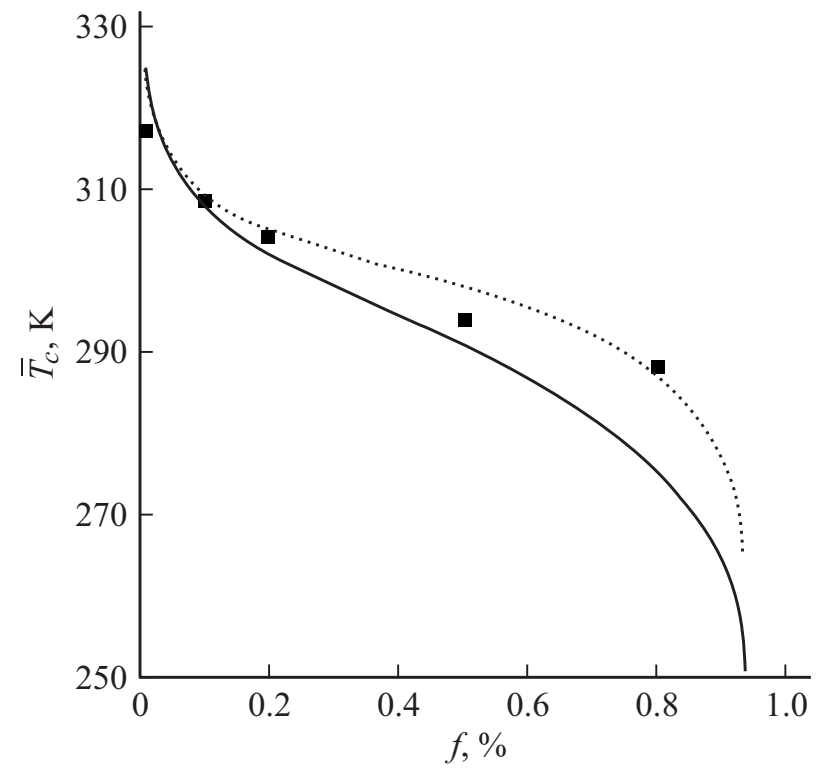

Рис. 5. Изменение усредненной температуры $\bar{T}_{c}=\left(T_{c}^{M}+T_{c}^{A}\right)$ равного объема фаз при прямом и обратном мартенситных переходах в сплаве $\mathrm{Ni}-\mathrm{Mn}-\mathrm{Ga}-\mathrm{Tb}$ с ростом объемной концентрации $f$ частиц Тb [1]. Кривая - расчет согласно уравнениям (7) и (8), пунктир - зависимость $\bar{T}_{c}(f)$ в отсутствие микронапряжений $\left(\delta_{f}=0\right)$.

ческого размера преципитатов $d_{k}$ в сплаве $\mathrm{TiNi}$ можно предположить существование критической объемной концентрации $f_{k}$ частиц Ть интервале $1 \%>f>0.8 \%$, выше которой мартенситного превращения в сплаве $\mathrm{NiMnGaTb}$ не происходит.

На рис. 6 приведена зависимость усредненного значения температурного интервала $\Delta T=\left(\Delta T^{M}+\Delta T^{A}\right) / 2$ прямого, $\Delta T^{M}=M_{s}-M_{f}$, и обратного, $\Delta T^{A}=A_{f}-A_{s}$, мартенситных переходов в сплаве $\mathrm{NiMnGaTb}$ от объемной концентрации частиц Tb. Обращает на себя внимание приблизительно линейный характер этой зависимости вплоть до $f=0.8 \%$. Согласно [1] выделения тербия когерентно связаны с матрицей; несовместность деформации между матрицей и преципитатом составляет $\delta_{0}=10 \%$. Она служит источником внутренних напряжений в сплаве. Линейный характер зависимости температурного интервала перехода от концентрации частиц тербия свидетельствует о том, что ширина перехода по температуре определяется в данном случае не локальным упругим полем частиц (как это было в рассмотренном выше сплаве $\mathrm{TiNi}$, a распределенным полем упругих деформаций $\varepsilon_{e}=\delta_{0} f$. Согласно Эшелби $[12,13]$ это поле создает распределенное поле упругих напряжений

$$
\sigma_{e}(f)=\left(\frac{1-v}{1+v}\right) \frac{\delta_{0} E}{1-2 v} f .
$$

Подставляя энергию упругих напряжений $W_{e}(f)=$ $=\varepsilon_{m} \sigma_{e}(f)$ в соотношения (3), получим зависимость температур $T_{c}^{M}$ и $T_{c}^{A}$, ширины перехода $\Delta T$ и кинетического фактора $k_{0}(f)$ от концентрации частиц тербия,

$$
\begin{aligned}
& T_{c}^{M}(f)=\left[1-\delta_{f} f+\frac{k_{\mathrm{B}} T_{c 0}}{q \omega(d, f)} \ln \left[\frac{3}{2} k_{0}(d, f)-1\right]\right] T_{c 0}, \\
& T_{c}^{A}(f)=\left[1+\frac{k_{\mathrm{B}} T_{c 0}}{q \omega(d, f)} \ln \left[3 k_{0}(d, f)-1\right]\right] T_{c 0}, \\
& \Delta T(f)=\Delta T_{0} \\
& +\left[\delta_{f} f+\frac{k_{\mathrm{B}} T_{c 0}}{q \omega(d, f)} \ln \left(\frac{3 k_{0}(d, f)-1}{3 k_{0}(d, f) / 2-1}\right)\right] T_{c 0}, \\
& k_{0(d, f)}=\left(\frac{l_{p}}{d_{c}}\right)^{2}=\left(\frac{d}{d_{c}} \beta R(f)\right)^{2}, \\
& \delta_{f}=\left(\frac{1-v}{1+v}\right) \frac{\varepsilon_{m} \delta_{0} E}{(1-2 v) q},
\end{aligned}
$$

где $\Delta T_{0}$ - ширина перехода в отсутствие наночастиц.

Авторы [1] полагают, что отсутствие мартенситного перехода при концентрации частиц $1 \%$ связано с тем, что расстояние между частицами $l_{p}$ становится величиной порядка поперечного размера частиц $d$. $\mathrm{B}$ действительности, при $\beta \approx 1$ (форма частиц близка к сферической) свободное расстояние между частицами $l_{p}(f)=R(f) d$ при $f=1 \%$ составляет $\approx 15 d$. Это означает, что имеется другой источник пространственного стеснения перемещения дислокаций фазового превращения. Таким источником является размер зерен $D$.

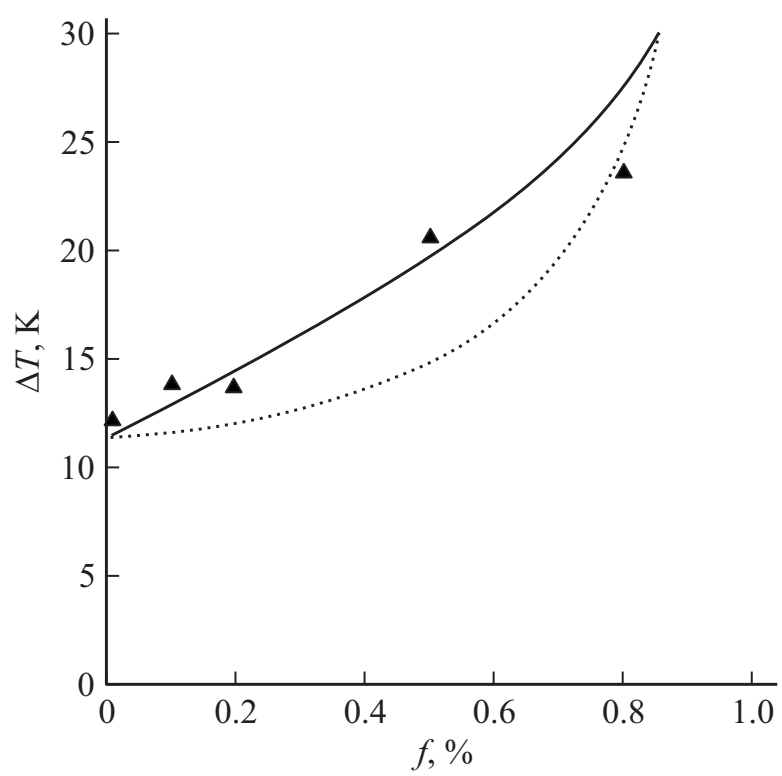

Рис. 6. Зависимость усредненного значения температурной ширины $\Delta T=\left(\Delta T^{M}+\Delta T^{A}\right) / 2$ прямого, $\Delta T^{M}=M_{s}-M_{f}$, и обратного, $\Delta T^{A}=A_{f}-A_{s}$, мартенситных переходов в сплаве $\mathrm{Ni}-\mathrm{Mn}-\mathrm{Ga}-\mathrm{Tb}$ от объемной концентрации $f$ частиц $\mathrm{Tb}[1]$. Кривая - расчет согласно уравнениям (7) и (8), пунктир зависимость $\Delta T(f)$ в отсутствие микронапряжений $\left(\delta_{f}=0\right)$. 


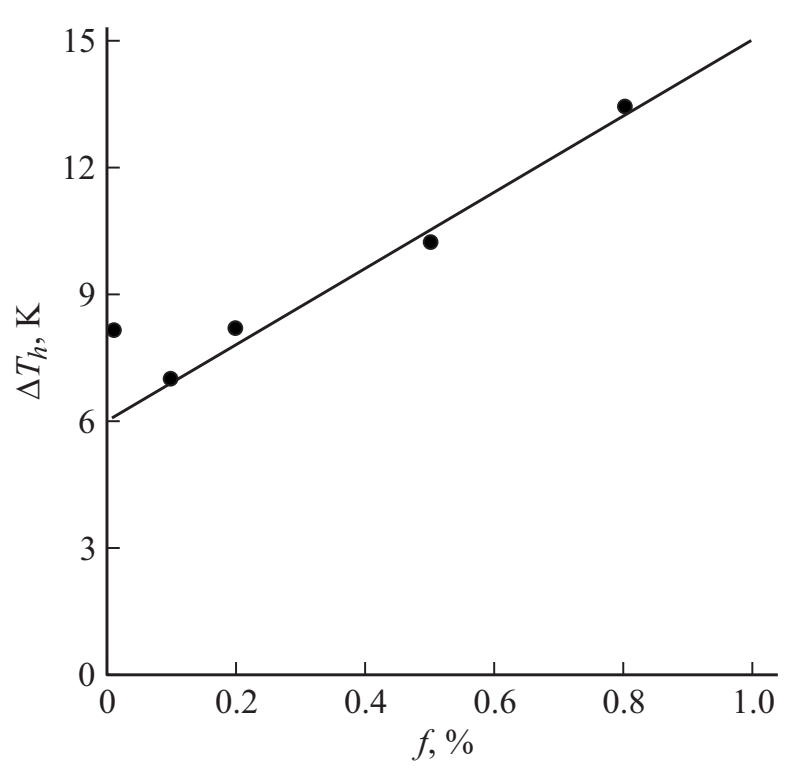

Рис. 7. Зависимость температурного гистерезиса мартенситного перехода в сплаве $\mathrm{Ni}-\mathrm{Mn}-\mathrm{Ga}-\mathrm{Tb}$ от объемной концентрации $f$ частиц $\mathrm{Tb}[1]$.

В отсутствие частиц он определяет величину элементарного объема превращения $\omega_{0}=\pi D^{2} a_{0} / 4$ и величину критического размера $d_{c}=D$ в соотношении (7d) для кинетического фактора. С учетом этого обстоятельства для элементарного объема превращения и кинетического фактора имеем следующие соотношения,

$$
\begin{gathered}
\frac{1}{\omega(d, f, D)}=\frac{1}{\omega_{0}}\left[1+\left(\frac{D}{\beta R(f) d}\right)^{2}\right], \\
k_{0}(d, f, D)=\left(\frac{d}{D} \beta R(f)\right)^{2} .
\end{gathered}
$$

На рис. 5 и 6 кривые демонстрирует результаты расчета согласно уравнениям (7) и (8) температуры $\bar{T}_{c}=\left(T_{c}^{M}+T_{c}^{A}\right) / 2$ и температурного интервала перехода $\Delta T$ от объемной концентрации $f$ частиц Тb. При расчете использовались следующие значения коэффициентов и параметров: $d=10 \mathrm{~nm}, D=176 \mathrm{~nm}$ (что близко к $D=215 \mathrm{~nm}[1]), \beta=1, \delta_{f}=5$ и $k T_{c 0} / \omega_{0} q=1 / 60$, $\Delta T_{0}=8 \mathrm{~K}$. На рис. 5 и 6 пунктирами обозначены эти зависимости согласно уравнениям (7) и (8) при $\delta_{f}=0$, т.е. в отсутствие когерентной связи частиц $\mathrm{Tb}$ с матрицей. Видно, что независимо от этого обстоятельства при концентрации частиц $f_{k} \approx 0.9 \%$ температура $\bar{T}_{c}$ устремляется к нулю, а температурная ширина перехода к неопределенно большой величине. Отметим также, что согласно данным [1] зависимость температурного гистерезиса превращения $\Delta T_{h}$ от концентрации частиц $f$ имеет линейный характер с наклоном, соответствующим величине коэффициента $\delta_{f}=3.1$ (рис. 7).

\section{4. Заключение}

Таким образом, произведенный в рамках теории размытых мартенситных переходов анализ влияния дисперсных наночастиц на параметры мартенситных переходов в сплавах с эффектом памяти формы позволяют сделать следующие выводы.

1. Наночастицы в сплавах с ЭПФ при когерентной связи частиц с матрицей являются источниками гетерогенного зарождения дислокаций фазового (мартенситного) превращения и возникновения распределенных упругих деформаций и микронапряжений; в отсутствие когерентной связи они служат источником формирования элементарного объема превращения из-за пространственного стеснения перемещения дислокаций превращения.

2. Частицы оказывают сильное влияние на термодинамические параметры мартенситных переходов в этих сплавах, такие как температура перехода, его ширина и гистерезис; и на кинетические параметры, такие как существование критических значений размера частиц и их объемной концентрации, соответственно, ниже и выше которых мартенситное превращение в сплаве блокируется.

3. В количественном отношении влияние наночастиц на термодинамические и кинетические параметры мартенситных переходов в сплавах с ЭПФ зависит от размера частиц и их объемной концентрации.

\section{Список литературы}

[1] Y. Wu, J. Wang, C. Jiang, H. Xu. Intermetallics 97, 42 (2018).

[2] X. Wang, S. Kustov, R. Li, D. Schryvers, B. Verlinden, J. Van Humbeeck. Acta Mater. 82, 224 (2015).

[3] Е.Ю. Панченко, Ю.И. Чумляков, И.В. Киреева, А.В Овсяников, Х. Сехитоглу, И. Караман, Г. Майер. ФММ 106, 597 (2008).

[4] W. Cai, J. Zhang, Z.Y. Gao, J.H. Sui. Appl. Phys. Lett. 92, 252502 (2008).

[5] X. Yi, X. Meng, W. Cai, L. Zhao. Scripta Mater. 151, 90 (2018).

[6] Г.А. Малыгин, В.И. Николаев, С.А. Пульнев. ЖТФ 89, N 7, 132 (2019).

[7] Г.А. Малыгин. УФН 171, 187 (2001).

[8] Г.А. Малыгин. ФТТ 61, 288 (2019).

[9] Г.А. Малыгин. ФТТ 61, 1310 (2019).

[10] K. Otsuka, X. Ren. Progr. Mater. Sci. 50, 511 (2005).

[11] Дж. Эшелби. Континуальная теория дислокаций. Изд-во ИЛ, М. (1963). 247 c.

[12] D.Y. Li, L.Q. Chen. Acta Mater. 45, 471 (1997).

[13] Г.А. Малыгин. ФТТ 45, 1491 (2003).

[14] T. Honma. Shape Memory Alloy-86 / Ed. Ch. Yoyi, T.Y. Hsu, T. Ko. China Academic Publ., Guilin. (1986). P. 47.

Редактор Т.Н. Василевская 